\title{
Liderazgo femenino: evidencia
}

\author{
Female leadership: evidence \\ Claudia Marcela Medina Monje* \\ *Ortopedista y Traumatóloga. Magister en Educación. IPS Universitaria sede Clínica León XIII. Medellín, Colombia.
}

\begin{abstract}
Resumen
Teniendo como base los tres componentes de la evidencia (lo que está publicado en la literatura, los valores y preferencias, y la experiencia) se revisan los factores diferenciadores en las habilidades y actitudes de liderazgo entre los roles de género asignados socialmente a una persona según sus características biológicas y que se codifican en hombre-mujer. La literatura reporta que hay más similitudes que diferencias en el liderazgo de hombres y mujeres; hay situaciones condicionadas al contexto y edad, y sólo se han reportado diferencias significativas en habilidad verbal, visoespacial y matemática. Pero, a la luz de las publicaciones basadas en metaanálisis, quedan desvirtuadas las afirmaciones acerca de las grandes diferencias en los estilos de liderazgo entre géneros. Los valores y preferencias de las personas se configuran a lo largo del tiempo según el contexto en el que viven, y genera que ciertas ideas y percepciones sean privilegiadas, dando a su vez voz a estereotipos sobre el liderazgo femenino y masculino, elementos que no están reflejados en los estudios publicados. Por último, la experiencia como tercer componente de la evidencia expone brevemente las vivencias personales de algunas líderes que están publicadas en la literatura.
\end{abstract}

Palabras clave: Liderazgo, habilidades blandas, liderazgo femenino, mujeres.

\begin{abstract}
Based on the three components of the evidence (what is published in the literature, the values and preferences and the experience), the differentiating factors in the leadership skills and attitudes between the gender roles socially assigned to a person according to their biological characteristics and that are encoded in male-female. The literature reports that there are more similarities than differences in the leadership of men and women; there are situations conditioned to the context and age, and only significant differences have been reported in verbal, visuospatial and mathematical ability. But, in the light of publications based on meta-analysis, claims about large differences in leadership styles between genders are disproved. The values and preferences of people are configured over time according to the context in which they live and generates that certain ideas and perceptions are privileged, giving voice to stereotypes about female and male leadership, elements that are not reflected in published studies. Finally, the experience as the third component of the evidence, briefly exposes the personal experiences of some leaders that are published in the literature.
\end{abstract}

Keywords: Leadership, soft skills, female leadership, women.

\section{Introducción}

Tradicionalmente se han reproducido estereotipos sobre las condiciones y características de liderazgo de los diferentes géneros. Investigaciones publicadas en los años 70 reportaban diferencias en términos de comportamientos, mas no de estilos, comportamientos influenciados por las presiones sociales de la época. ${ }^{1}$

\section{Correspondencia:}

Claudia Marcela Medina Monje

E-mail: cmedinamonje@gmail.com

Recibido: 12-11-2021. Aceptado: 22-11-2021.
Un tema que ha sido recurrente acerca de los estilos de liderazgo es su clasificación como «femeninos» 0 «masculinos» de acuerdo con la utilización de ciertos atributos que le son asignados. ${ }^{2}$

Hace ya varios años se ha venido hablando de la habilidad que tienen las mujeres de ejercer un liderazgo conectivo, que es un modelo de liderazgo integrador mucho más adecuado para el dramáticamente cambiante lugar de trabajo del siglo XXI. Estas
Citar como: Medina MCM. Liderazgo femenino: evidencia. Orthotips. 2022; 18 (1): 64-67. https://dx.doi.org/10.35366/103734 
habilidades sociales y de conexión, probablemente hace unas pocas décadas, eran ocultadas por las líderes femeninas con el fin de garantizar su permanencia y éxito en las condiciones de la época. ${ }^{3}$

Es común en las publicaciones de estilos de liderazgo y comportamiento organizacional mencionar que las mujeres tienen un estilo más "democrático» para liderar compartiendo poder y pensamientos, mientras que los hombres son más «directivos» en su estilo para liderar. ${ }^{4}$

\section{Literatura}

En el entorno popular, es ampliamente extendido el concepto de que los hombres y mujeres son psicológicamente muy diferentes y eso se traduce en características de liderazgo muy diferentes. Un solo ejemplo de ello es el libro Los hombres son de Marte, las mujeres son de Venus, que ha vendido más de 30 millones de copias y ha sido traducido a más de 40 idiomas y que en sus páginas expone enormes diferencias psicológicas entre mujeres y hombres. ${ }^{5}$

Hay reseñas de investigaciones acerca de diferencias psicológicas de género desde inicios del siglo XX. En 1974 se publicó el libro The psychology of sex differences, ${ }^{6}$ que revisó más de 2,000 estudios de diferencias de género en una amplia variedad de dominios: habilidades, personalidad, comportamiento social y memoria; con los hallazgos se desestimaron muchas creencias populares, como que las mujeres son más sociales, más sugestionables, que tienen menos autoestima y que son mejores en memorizar y en hacer tareas simples. Concluyeron, según su revisión, que había diferencias en habilidad verbal, visoespacial, matemática y agresión. ${ }^{5}$

Una revisión sistemática publicada en el 2005 en el Journal American Psychologist revisó 26 metaanálisis, con los que soportó la hipótesis de las similitudes entre los géneros. Las diferencias entre los géneros pueden variar sustancialmente en magnitud, en diferentes edades y contextos. Las afirmaciones exageradas de diferencias de estilos de liderazgo entre los diferentes géneros conllevan costos sustanciales en los lugares de trabajo y en las relaciones. Según este estudio, en términos estadísticos, la mayor parte de las diferencias psicológicas entre los géneros están cercanas a cero. ${ }^{5}$

Ideas como que la mujer prioriza la familia sobre la carrera, no es buena negociando, tiene poca confianza en sí misma o le teme a asumir riesgos, han sido desmitificadas en los últimos años gracias a metaanálisis que han demostrado que hombres y mujeres tienen similares inclinaciones, actitudes y habilidades. ${ }^{7}$

La mayoría de los líderes, independientemente de que sean hombres o mujeres, exhiben unas características comunes como: buenas relaciones personales, inteligencia, confianza y habilidad social, entre otras; tal vez por esta razón no hay diferencias significativas en los estudios. ${ }^{8}$

\section{Valores y preferencias}

Gran parte de las publicaciones del tema de diferencias en estilos de liderazgo, según el género, provienen de opiniones y percepciones de las personas. En 2019, Harvard Business Review publicó Women score higher than men in most leadership skills, en donde según lo reportado, analizaron «miles» de evaluaciones en 360 grados, reportaron que las mujeres son percibidas tan efectivas como los hombres en los puestos de liderazgo; asimismo, fueron calificadas como sobresalientes en toma de iniciativa, actuar con resiliencia, practicar el autodesarrollo, impulsar resultados y mostrar alta integridad y honestidad. También se reporta que las mujeres no son tan generosas autoevaluándose como los hombres; la publicación concluye anotando que a las mujeres no las detiene la falta de capacidad, sino la falta de oportunidades, muchas de ellas ocasionadas por prejuicios culturales y la eliminación muy lenta de los estereotipos. ${ }^{9}$

Si bien,la publicación muestra algunas diferencias en los porcentajes en la mayor parte de habilidades (84\%) a favor de las mujeres, esta diferencia es del $2 \%$ o menos. Se requeriría un análisis estadístico completo para hacer afirmaciones del significado de los hallazgos.

\section{Experiencia}

En una publicación reciente de JAMA, entrevistaron a 20 mujeres líderes del área quirúrgica, investigando acerca de los puntos que, ellas consideran, contribuyeron a su crecimiento personal. Destacaron como factores internos que influyeron algunos rasgos de personalidad como la confianza, la resiliencia y el desinterés en la recompensa; la adaptabilidad fue descrita como el mayor facilitador para el éxito en sus carreras, además como factor externo, el tener mentores de todos los géneros que impulsaron sus carreras. ${ }^{10}$ 
Mi experiencia en posiciones de liderazgo, en las que sigo aprendiendo todos los días, me ha mostrado la relevancia de tener mentores (hombres y mujeres); tener clara la diferencia entre un gestor y un líder, siendo el líder una persona al servicio de los demás y con el propósito de marcar diferencias positivas para todos los integrantes de su equipo, impactando, influenciando e inspirando. Cada acción personal que se haga aporta en generar el cambio cultural que se requiere para que naturalicemos, que quien esté en la posición de líder sea una persona capaz y comprometida, independientemente del género. Debemos contribuir a hacernos visibles entre nosotras sin poner por encima nuestra condición de mujeres para conseguirlo, somos cada vez más notorias porque tenemos las habilidades y capacidades $y$, finalmente, si de equidad, diversidad e inclusión estamos hablando, por supuesto que esas habilidades de liderazgo deben beneficiar a todas las personas a nuestro alrededor, independientemente de su género.

\section{Discusión}

En los últimos años se ha trabajado en disminuir la brecha que existe en posiciones de liderazgo para las mujeres; precisamente esas posiciones son un paso más para impulsar la paridad de género. ${ }^{11}$

Cabe anotar que en algunos países, como Estados Unidos, se ha encontrado una fuerte correlación entre la cantidad de mujeres miembros de una sociedad y la proporción de miembros de las juntas directivas de las mismas, pero, aún faltan más miembros jóvenes motivadas a participar. ${ }^{12}$

Implementar estrategias de mentoría, evitar el aislamiento social y profesional, y mejorar las estrategias de reclutamiento, retención y promoción nos acercarán cada vez más a una mayor presencia de mujeres en la especialidad y en las posiciones de liderazgo. ${ }^{13}$

Se debe propender por crear modelos a seguir más competentes que permitan a hombres y mujeres avanzar en sus carreras. ${ }^{14}$

Sin desconocer la brecha actual en las posiciones de liderazgo, la intención en este artículo fue revisar la evidencia existente en las diferencias de estilos de liderazgo entre hombres y mujeres. No estamos tan distantes unos de otros de acuerdo con los estudios publicados y con las percepciones de las personas.
Se debería normalizar el hecho de que en algún momento pueda haber más mujeres que hombres en posiciones de liderazgo en las organizaciones, pero esto no está motivado por ser mujer, es porque cada vez hay más visibilidad, procesos más transparentes, mentoría y modelos a seguir que están impulsando a mujeres muy capaces que hasta hace unos años no tenían la oportunidad de participar.

Hay mucha atención en la decisión de compensar la disparidad existente en la presencia de mujeres en la especialidad de ortopedia. Es el momento de empezar a revisar si en nuestros países hay diferencias en los estilos de liderazgo entre hombres y mujeres en medicina, no para competir pensando en quién es mejor, sino para producir sinergias que van a redundar en beneficios para todos, independientemente del género.

La mayor parte de líderes, independientemente de que sean hombres o mujeres, exhiben unas características comunes como buenas relaciones personales, inteligencia, confianza y habilidad social, entre otras, tal vez por esta razón no hay diferencias significativas en los estudios. ${ }^{8}$

\section{Conclusiones}

El desempeño del liderazgo incluye habilidades duras y blandas, muchas de ellas difíciles de medir cuantitativamente. Por lo que el desempeño de éstas se define basándose en las percepciones de las personas.

Se debe considerar el costo laboral y personal que generan las afirmaciones sin fundamento científico acerca de las diferencias de género. Éstas se ven representadas en pérdida de oportunidades tanto para hombres como para mujeres.

Es importante que quienes tengan la responsabilidad y privilegio de seleccionar personas en el entorno laboral o académico sean conscientes de los estereotipos o sesgos que tengan.

Debe haber líneas de investigación que busquen obtener información de los mejores potenciales en los diferentes estilos de liderazgo y generar espacios donde haya sinergia.

\section{Referencias}

1. Chapman JB. Comparison of male and female leadership styles. Acad Manage J. 1975; 18 (3): 645-650.

2. Farrugia G, Zorn CK, Williams AW, Ledger KK. A qualitative analysis of career advice given to women leaders in an academic medical center. JAMA Netw Open. 2020; 3 (7): e2011292. 
3. Lipman-Blumen J. Connective leadership: Female leadership styles in the 21st-century workplace. Sociol Perspect. 1992; 35 (1): 183-203.

4. Robbins SP. Organizational behavior: Concepts, controversies and applications. 7th ed. London, England: Prentice-Hall; 1996.

5. Hyde JS. The gender similarities hypothesis. Am Psychol. 2005; 60 (6): 581-592.

6. Maccoby EE, Jacklin CN. The psychology of sex differences. Palo Alto, CA, Estados Unidos de América: Stanford University Press; 1975.

7. Tinsley $\mathrm{CH}$, Ely RJ. What most companies get wrong about men and women. Harvard Business Review [Internet]. May 01, 2018 [cited Nov 21, 2021]. Available in: https://hbr.org/2018/05/ what-most-people-get-wrong-about-men-and-women

8. Rahman MA. Influence of styles of women leadership and organization culture on locus of control and job satisfaction of Uin Alauddin Makassar Lecturer. IJBE. 2018; 2 (2): 1-13.

9. Zenger J, Folkman J. Research: women score higher than men in most leadership skills. Harvard Business Review [Internet]. June 25, 2019 [cited Nov 21, 2021]. Available in: https://hbr. org/2019/06/research-women-score-higher-than-men-in-mostleadership-skills

10. Columbus AB, Lu PW, Hill SS, Fields AC, Davids JS, Melnitchouk N. Factors associated with the professional success of female surgical department chairs: a qualitative study. JAMA Surg. 2020; 155 (11): 1028-1033.

11. Hiemstra LA, Wittman T, Mulpuri K, Vezina C, Kerslake S. Dissecting disparity: improvements towards gender parity in leadership and on the podium within the Canadian Orthopaedic Association. J ISAKOS. 2019; 4 (5): 227-232.

12. Saxena S, Cannada LK, Weiss JM. Does the proportion of women in orthopaedic leadership roles reflect the gender composition of specialty societies? Clin Orthop Relat Res. 2020; 478 (7): 1572-1579.

13. Joyce AC. Perspectives of women in orthopaedic surgery on leadership development [Graduate Theses and Dissertations]. USA: University of South Florida; 2016. Available in: https://digitalcommons.usf.edu/cgi/viewcontent. cgi?article $=7718 \&$ context $=$ etd

14. Chamorro-Premuzic T, Gallop C. 7 leadership lessons men can learn from women. Harvard Business Review [Internet]. April 01, 2020 [cited Nov 21, 2021]. Available in: https://hbr. org/2020/04/7-leadership-lessons-men-can-learn-from-women

\section{Conflicto de intereses}

No tengo conflicto de intereses para declarar. 